\title{
A generalized model for the effective thermal conductivity of porous media based on self-similarity
}

\author{
Yongjin Feng ${ }^{1}$, Boming Yu ${ }^{1,2,3}$, Mingqing $\mathrm{Zou}^{1}$ and \\ Duanming Zhang ${ }^{1}$ \\ ${ }^{1}$ Department of Physics and the State Key Laboratory of Laser Technology, Huazhong \\ University of Science and Technology, 1037 Luoyu Road, Wuhan 430074, People's Republic \\ of China \\ ${ }^{2}$ The State Key Laboratory of Nonlinear Mechanics (LNM), Institute of Mechanics, Chinese \\ Academy of Science, Beijing, 100080, People's Republic of China
}

E-mail: yu3838@public.wh.hb.cn

Received 20 May 2004, in final form 20 August 2004

Published 8 October 2004

Online at stacks.iop.org/JPhysD/37/3030

doi: $10.1088 / 0022-3727 / 37 / 21 / 014$

\begin{abstract}
A generalized model for the effective thermal conductivity of porous media is derived based on the fact that statistical self-similarity exists in porous media. The proposed model assumes that porous media consist of two portions: randomly distributed non-touching particles and self-similarly distributed particles contacting each other with resistance. The latter are simulated by Sierpinski carpets with side length $L=13$ and cutout size $C=3,5,7$ and 9, respectively, depending upon the porosity concerned. Recursive formulae are presented and expressed as a function of porosity, ratio of areas, ratio of component thermal conductivities and contact resistance, and there is no empirical constant and every parameter has a clear physical meaning. The model predictions are compared with the existing experimental data, and good agreement is found in a wide range of porosity of $0.14-0.80$, and this verifies the validity of the proposed model.
\end{abstract}

\section{Introduction}

The effective thermal conductivities of various kinds of porous media, such as granular materials, dispersed spheres, fibrous composites and packed beds, have received continuous attention [1-13] due to their various applications in science and engineering. In the past, many models for the thermal conductivity of porous media were proposed.

Krupiczka [2] approximated a packed bed as a bundle of long cylinders and numerically solved a set of two heat conduction equations in two dimensions with perfect contact at the solid-fluid interface. He then extended the results to a spherical lattice and proposed a correlation for the dimensionless effective thermal conductivity, $k_{\mathrm{e}}^{+}$:

$$
k_{\mathrm{e}}^{+}=\frac{k_{\mathrm{e}}}{k_{\mathrm{f}}}=(\beta)^{0.280-0.757 \log \varphi-0.057 \log \beta},
$$

where $k_{\mathrm{e}}$ and $k_{\mathrm{f}}$ are the effective thermal conductivity and the thermal conductivity of the fluid (matrix), respectively, $\beta=k_{\mathrm{s}} / k_{\mathrm{f}}$ is the ratio of the solid thermal conductivity to the fluid thermal conductivity and $\varphi$ is the porosity.

Zehnder and Schlunder [3] proposed a correlation for the stagnant thermal conductivity based on a one-dimensional heat flow model for heat conduction through a packed bed of spherical particles, the correlation for the dimensionless effective thermal conductivity, $k_{\mathrm{e}}^{+}$, being expressed as

$$
\begin{aligned}
k_{\mathrm{e}}^{+}= & 1-(1-\varphi)^{1 / 2}+\frac{2(1-\varphi)^{1 / 2}}{1-B / \beta} \\
& \times\left[\frac{(1-1 / \beta) B}{(1-B / \beta)^{2}} \ln \frac{\beta}{B}-\frac{B+1}{2}-\frac{B-1}{1-B / \beta}\right],
\end{aligned}
$$

where the shape factor, $B$, is approximated by

$$
B=1.25\left(\frac{1-\varphi}{\varphi}\right)^{10 / 9} \text {. }
$$

3 Author to whom any correspondence should be addressed. 
Compared with the experimental results of Nozad et al [6] for a packed-sphere bed, it is found that equations (1) and (2) underpredict the stagnant thermal conductivity when the ratio of the solid to fluid conductivity is high (see the comparison by Ma et al [13]). Furthermore, equations (1) and (2) contain several empirical constants with no physical meaning, and the roles of microstructural parameters in thermal conductivity are thus ignored. A comprehensive review of empirical correlations for thermal conductivity of porous media is given by Kaviany [11].

Hsu et al [10] developed a lumped-parameter model for the effective stagnant thermal conductivity of some two-dimensional and three-dimensional spatially periodic media. The geometries under investigation include arrays of touching and non-touching in-line squares and cylinders (two-dimensional), as well as touching and non-touching in-line cubes (three-dimensional). The dimensionless effective stagnant thermal conductivity, $k_{\mathrm{e}}^{+}$, for three-dimensional cubes with thermal resistance is expressed as

$$
\begin{aligned}
k_{\mathrm{e}}^{+}= & 1-\gamma_{\mathrm{a}}^{2}-2 \gamma_{\mathrm{a}} \gamma_{\mathrm{c}}+2 \gamma_{\mathrm{a}}^{2} \gamma_{\mathrm{c}}+\frac{\gamma_{\mathrm{c}}^{2} \gamma_{\mathrm{a}}^{2}}{1 / \beta} \\
& +\frac{\gamma_{\mathrm{a}}^{2}-\gamma_{\mathrm{a}}^{2} \gamma_{\mathrm{c}}^{2}}{1-\gamma_{\mathrm{a}}+\gamma_{\mathrm{a}} / \beta}+\frac{2\left(\gamma_{\mathrm{a}} \gamma_{\mathrm{c}}-\gamma_{\mathrm{a}}^{2} \gamma_{\mathrm{c}}\right)}{1-\gamma_{\mathrm{a}} \gamma_{\mathrm{c}}+\gamma_{\mathrm{a}} \gamma_{\mathrm{c}} / \beta}
\end{aligned}
$$

and the porosity, $\varphi$, is related to the geometrical parameters $\left(\gamma_{\mathrm{a}}\right.$ and $\left.\gamma_{\mathrm{c}}\right)$ by

$$
1-\varphi=\left(1-3 \gamma_{c}^{2}\right) \gamma_{a}^{3}+3 \gamma_{c}^{2} \gamma_{a}^{2} .
$$

Equation $(3 b)$ is obtained by geometrical consideration. A comparison of the results based on equation (3) with existing experimental data shows that they are in excellent agreement with the experimental data if the contact resistance parameter $\gamma_{\mathrm{c}}=0.13$ is chosen at a porosity of 0.36 . However, no comparison with experimental data at different porosities was given. In fact, the two parameters, $\gamma_{\mathrm{a}}$ and $\gamma_{\mathrm{c}}$, are related to the porosity through equation $(3 b)$, a nonlinear equation. It appears that the two parameters, $\gamma_{\mathrm{a}}$ and $\gamma_{\mathrm{c}}$, are a function of porosity, but no functional dependence was reported.

$\mathrm{Yu}$ and Cheng [12] developed a fractal thermal conductivity model for both mono- and bi-dispersed porous media by assuming that porous media consist of two portions: some particles contact each other to form tortuous chains, whereas others do not touch each other (non-touching). Their fractal thermal conductivity model for mono-dispersed porous media (corresponding to packed beds) is

$$
\begin{aligned}
k_{\mathrm{e}}^{+}= & \frac{A_{\mathrm{nt}}}{A}\left[(1-\sqrt{1-\varphi})+\frac{\sqrt{1-\varphi}}{1+(1 / \beta-1) \sqrt{1-\varphi}}\right] \\
& +\left(1-\frac{A_{\mathrm{nt}}}{A}\right) \frac{\lambda_{\mathrm{p}, \max }^{2}}{A}\left(\frac{\lambda_{\mathrm{p}, \max }}{L_{0}}\right)^{D_{\mathrm{T}}-1} \frac{D_{\mathrm{f}}}{1+D_{\mathrm{T}}-D_{\mathrm{f}}} \\
& \times\left[\frac{\gamma_{\mathrm{a} 1}}{\beta}+\frac{1-\gamma_{\mathrm{a} 1}}{\gamma_{\mathrm{c} 1}^{2}(\beta-1) / \gamma_{\mathrm{a} 1}^{2}+1}\right]^{-1},
\end{aligned}
$$

where $A_{\mathrm{nt}}\left(0 \leqslant A_{\mathrm{nt}} / A \leqslant 1, A\right.$ is the total area of a unit cell) is an equivalent area of a cross section having the same porosity as the non-touching particles, $\gamma_{\mathrm{a} 1}$ is the ratio of the geometrical length scales, $\gamma_{\mathrm{c} 1}$ is the ratio of the contact length scales, $\lambda_{\mathrm{p}, \max } / L_{0}$ represents the ratio of the maximum particle diameter $\left(\lambda_{\mathrm{p}, \max }\right)$ to the representative length $\left(L_{0}\right)$, and $D_{\mathrm{f}}$ and $D_{\mathrm{T}}$ are the fractal dimensions for particles or pores and for tortuous particle/cluster chains, respectively. The term
$A_{\mathrm{nt}} / A[(1-\sqrt{1-\varphi})+(\sqrt{1-\varphi}) /(1+(1 / \beta-1) \sqrt{1-\varphi})]$ in equation (4) represents the contribution from non-touching particles [10]. However, this fractal model contains as many as seven parameters, $A_{\mathrm{nt}} / A, \gamma_{\mathrm{a} 1}, \gamma_{\mathrm{c} 1}, \lambda_{\mathrm{p}, \max } / L_{0}, \lambda_{\mathrm{p}, \max }^{2} / A, D_{\mathrm{f}}$ and $D_{\mathrm{T}}$, that have to be determined, although each parameter has a clear physical meaning.

Recently, Ma et al [13] have developed a self-similarity model for the effective thermal conductivity of porous media based on the thermal-electrical analogy technique and on the statistical self-similarity existing in porous media. The dimensionless effective thermal conductivity, $k_{\mathrm{e}}^{+}$is expressed as a function of the porosity, $\varphi$ (related to stage $n$ of the Sierpinski carpet), ratio of areas $\left(A_{\mathrm{nt}} / A\right)$, ratio $(\beta)$ of component thermal conductivities, and dimensionless contact resistance/width, $t^{+}$. The dimensionless effective thermal conductivity, $k_{\mathrm{e}}^{+}$, is

$$
\begin{aligned}
k_{\mathrm{e}}^{+}= & \frac{k_{\mathrm{e}}}{k_{\mathrm{m}}}=\frac{A_{\mathrm{nt}}}{A} k_{\mathrm{e}, \mathrm{nt}}^{+}+\left(1-\frac{A_{\mathrm{n}}}{A}\right) k_{\mathrm{e}, \mathrm{sc}}^{+}=\frac{A_{\mathrm{nt}}}{A}[(1-\sqrt{1-\varphi}) \\
& \left.+\frac{\sqrt{1-\varphi}}{1+(1 / \beta-1) \sqrt{1-\varphi}}\right]+\left(1-\frac{A_{\mathrm{nt}}}{A}\right) k_{\mathrm{e}, \mathrm{sc}}^{+(n)}
\end{aligned}
$$

and

$$
k_{\mathrm{e}, \mathrm{sc}}^{+(n)}=\frac{3 k_{\mathrm{e}, \mathrm{sc}}^{+(n-1)}}{2 /\left[t^{+} \beta^{(n)}+\left(1-t^{+}\right)\right]+1 /\left(2 / 3+\beta^{(n)} / 3\right)},
$$

where $\beta^{(n)}=\beta^{(0)} / k_{\mathrm{e}, \mathrm{sc}}^{+(n-1)}, A_{\mathrm{nt}} / A=0.55$ and the dimensionless contact resistance/width $t^{+}=0.00033$. In equation $(5 b)$, the superscript $n=1,2,3, \ldots$ represents the stage of the Sierpinski carpet, and $n=0$ refers to the zero-stage Sierpinski carpet (i.e. generator) and the subscript 'sc' represents the Sierpinski carpet. The effective thermal conductivity for the zero-stage Sierpinski carpet is

$$
k_{\mathrm{e}, \mathrm{sc}}^{+(0)}=\left[\frac{2}{3\left(t^{+} \beta^{(0)}+1-t^{+}\right)}+\frac{1}{2+\beta^{(0)}}\right]^{-1} .
$$

In equation (5), $\beta^{(0)}=k_{\mathrm{s}} / k_{\mathrm{f}}$ is the ratio of the solid conductivity to the fluid (matrix) conductivity. The porosity, $\varphi$, is related to the stage, $n$, of the Sierpinski carpet by

$$
\varphi=\left(\frac{8}{9}\right)^{n+1}
$$

Although every parameter in this model has a clear physical meaning and this model has the least parameters $\left(A_{\mathrm{nt}} / A\right.$ and $\left.t^{+}\right)$ compared with the other models, this model may be only applicable to the porosity range of about $0.3-0.5$ and fractal dimension $D_{\mathrm{f}}=1.89$. This means that if the porosity is in this range, the Sierpinski carpet with fractal dimension $D_{\mathrm{f}}=1.89$ can be approximately used to describe the porous medium. If, however, the porosity deviates much from this range, Sierpinski carpets with different fractal dimensions and different porosities may need to be constructed to simulate such media.

This work attempts to extend Ma et al's work [13] to a generalized model to cover a wide range of porosity of 0.14-0.80. For this purpose, Sierpinski carpets with different microstructures and different fractal dimensions are constructed to simulate the real porous media. Then, a generalized model for the effective thermal conductivity of porous media is derived. To be verified, the model predictions are finally compared with the existing experimental data. 


\section{Fractal description of porous media}

Euclidean geometry describes ordered objects such as points, curves, surfaces and cubes using integer dimensions $0,1,2$ and 3 , respectively. Their measures are invariant with respect to the unit of measurement used. However, numerous objects found in nature [14], such as rough surfaces, coast lines, mountains, rivers, lakes and islands, are disordered and irregular, and they do not follow the Euclidean description due to the scaledependent measures of length, area and volume. These objects are called fractals, and the dimensions of such objects are nonintegral and called Hausdorff dimensions, or simply fractal dimensions. Geometry structures such as the Sierpinski gasket, Sierpinski carpet and Koch curve are examples of exactly selfsimilar fractals or regular fractals, which exhibit self-similarity over an infinite range of length scales. Their dimensions are also called similarity dimensions [15]. However, exactly selfsimilar fractals in a global sense are rarely found in nature. Many objects found in nature are not exactly self-similar, such as coastlines, islands on earth, they are statistically self-similar and they are called statistical fractals. These objects exhibit self-similarity in some average sense and over a certain local range of length scales, $L$. The fractal dimensions used in this paper are applicable to both exactly self-similar fractals (such as the Sierpinski gasket, Sierpinski carpet and Koch curve) and statistical fractals (such as fractal/random porous media).

The measure of a fractal object, $M(L)$, is related to the length scale, $L$, through a scaling law in the form $[14,15]$

$$
M(L) \sim L^{D_{\mathrm{f}}},
$$

where $M$ can be the length of a line or the area of a surface or the volume of a cube or the mass of an object and $D_{\mathrm{f}}$ is the fractal dimension of an object. Equation (6) implies the property of self-similarity, which means that the value of $D_{\mathrm{f}}$ from equation (6) is a constant over a range of length scales, $L$.

Porous media such as soil, sandstones in an oil reservoir, packed beds in chemical engineering, fabrics used in liquid composite moulding and wicks in heat pipes consist of numerous irregular pores of different sizes spanning several orders of magnitude in length scales. The pore microstructures, both the pore size and the pore-interfaces, of such porous media exhibit fractal characteristics [16-25], and these media all follow the fractal power law equation (6) and these media are thus called fractal porous media.

Katz and Thompson [16] used scanning electron microscopy and optical data to show that the pore spaces of several sandstone samples are fractals and are self-similar over three to four orders of magnitude in length extending from $10 \AA$ to over $100 \mu \mathrm{m}$. They used fractal statistics to predict the correct porosity and obtained the following correlation

$$
\varphi=c\left(\frac{l_{1}}{l_{2}}\right)^{3-D_{\mathrm{f}}},
$$

where $D_{\mathrm{f}}(=2-3)$ is the fractal dimension of pores in three dimensions, $c$ is a constant of the order of 1 and $l_{1}$ and $l_{2}$ are the lower and upper limits of self-similar regions, respectively. Their experimental results show that the pore fractal dimension increases with porosity. This implies that the pore fractal dimension is scale dependent for real porous media, which is (a)

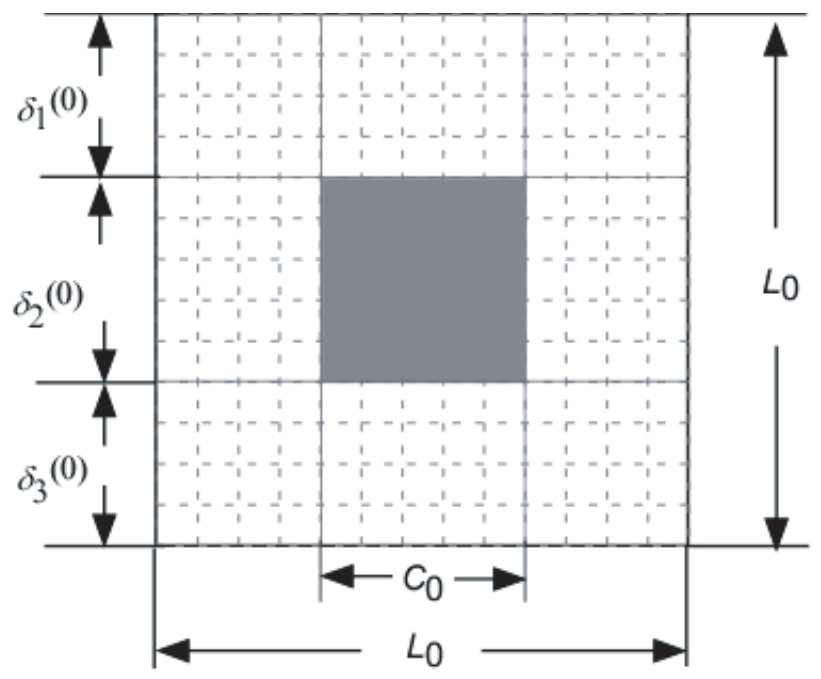

(b)

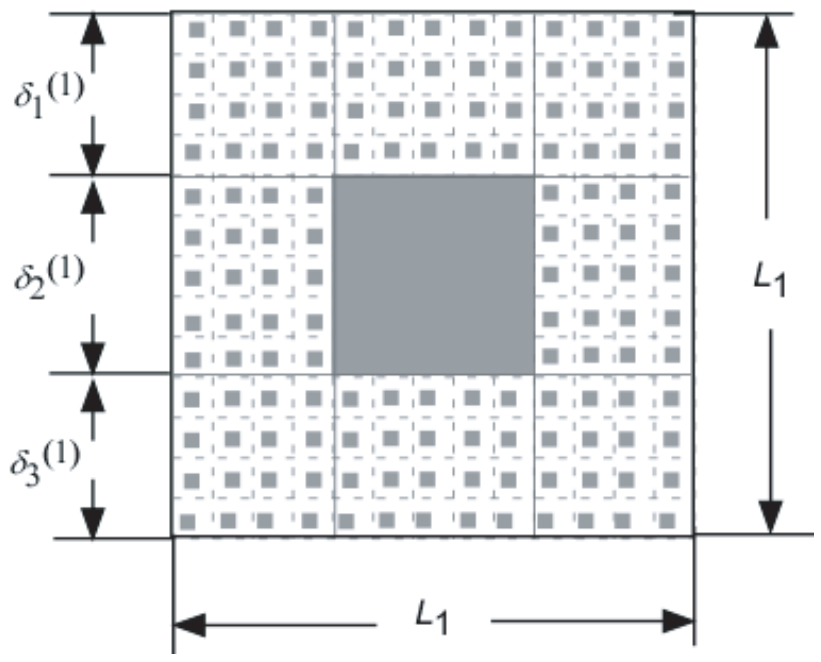

Figure 1. Sierpinski carpets with $L=13$ and $C=5$ and division of regions: $(a)$ zero-stage Sierpinski carpet and $(b)$ one-stage Sierpinski carpet.

different from exact self-similar fractals such as the Sierpinski carpet and the Sierpinski gasket, whose fractal dimension is constant at different porosities for a given structure, $C / L$, where $L$ and $C$ are the side length and cutout size, respectively. For example, the fractal dimension $D_{\mathrm{f}}=1.938$ and porosity $\varphi=144 / 169=0.85$ for a zero-stage Sierpinski carpet with $L=13$ and $C=5$ as shown in figure 1(a). For a one-stage Sierpinski carpet as shown in figure $1(b)$, the fractal dimension remains $D_{\mathrm{f}}=1.938$ (same as that of figure $1(a)$ ), but the porosity $\varphi=(144 / 169)^{2}=0.73$. Both the zero- and onestage carpets have the same structure, $C / L=5 / 13$, and the same fractal dimension $D_{\mathrm{f}}=1.938$. Therefore, in order to simulate real porous media, Sierpinski carpets with different microstructures and with different fractal dimensions should be constructed.

For both exactly self-similar fractal geometries (such as the Sierpinski carpet and Sierpinski gasket) and real porous media, Yu and Li [22] analytically derived a relation among the fractal dimension, porosity and scale for self-similarity in 


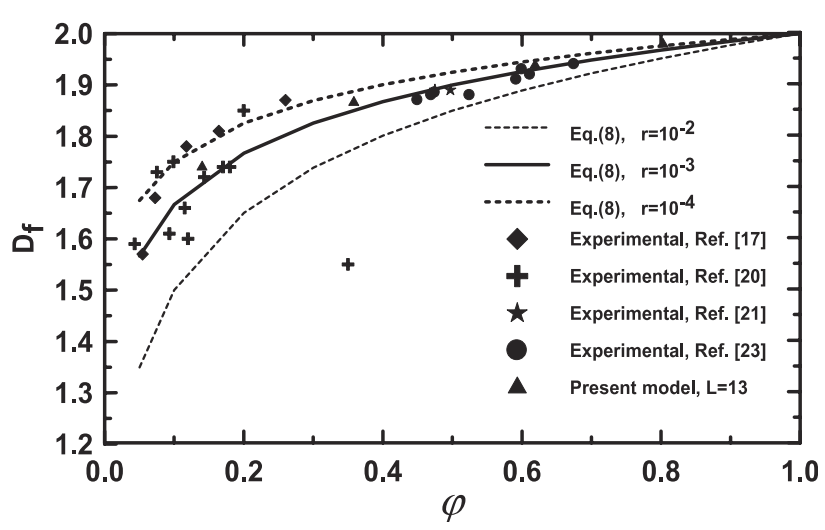

Figure 2. A summary for the pore area dimension, $D_{\mathrm{f}}$, versus porosity, $\varphi$, from the existing experimental data and for the results from equation (8) with different ratios $r=\lambda_{\min } / \lambda_{\max }$.

porous media, and the relation is given by

$$
D_{\mathrm{f}}=d-\frac{\ln \varphi}{\ln \left(\lambda_{\min } / \lambda_{\max }\right)},
$$

where $d$ is the Euclidean dimension, and $d=2$ and 3 in the two- and three-dimensional spaces, respectively. $\lambda_{\max }$ and $\lambda_{\min }$ are the maximum and minimum pore sizes in a unit cell for fractal porous media and are also the upper cutout and lower cutout scales in a self-similar medium. When $d=3$, equation (8) is identical with equation (7) as $c=1$ in equation (7). Equation (8) implies that the statistical self-similarity approximately exists in the range $\lambda_{\min }-\lambda_{\max }$. Equation (7) is applicable only to statistically self-similar fractal porous media because of the empiric constant $c$. Whereas equation (8) is applicable not only to exactly selfsimilar fractal geometries (such as the Sierpinski carpet and Sierpinski gasket, assuming $\lambda_{\min }=1$ and $\lambda_{\max }=$ side length of a carpet/gasket) but also to statistical self-similar fractal media (such as random/disordered porous media).

Figure 2 summarizes the results from the existing experimental measurements on fractal dimensions and porosities. The measurements show that fractal dimensions for real porous media increase with porosity, and the pore area fractal dimension will approach its possible maximum value of 2 as the porosity reaches the value of 1 . This is expected and is consistent with the physical situation. A rough fitting (see the solid line in figure 2) to these measurements by equation (8) gives the ratio $r=\lambda_{\min } / \lambda_{\max } \cong 10^{-3}$. Figure 2 or equation (8) with $r \cong 10^{-3}$ can be guidance for one to estimate the fractal dimension of a pore and to construct a proper Sierpinski carpet for modelling real porous media.

This section has presented a brief review of the description of fractal characters of porous media in nature, which form the theoretical bases of this work. For more evidence that porous media in nature are fractals, readers may consult the paper by Yu and Liu [25].

\section{Effective thermal conductivity based on statistical self-similarity}

To analyse the thermal conductivity of a porous medium, it is usually assumed that the medium has a periodic structure, and a unit cell or representative cell is then applied. Usually there are three approaches, Fourier's law models, Ohm's law models and empirical models, to predicting the thermal conductivity. Crane and Vachon [5] compared two approaches, Fourier's law models and Ohm's law models, for prediction of thermal conductivity. Ma et al [13] discussed briefly the three approaches and applied the Ohm's law model to develop a self-similarity model for the effective thermal conductivity of saturated porous media in the porosity range $0.30-0.50$. The present work applies the Ohm's law model approach and extends Ma et al's work to a generalized model for the effective thermal conductivity of porous media in a wide range of porosity, $0.14-0.80$, by constructing Sierpinski carpets with different fractal dimensions and different porosities, based on the assumption that the porous media consist of two portions: randomly distributed non-touching particles and self-similarly distributed particles contacting each other with thermal resistance.

To model the self-similarly distributed particles that contact each other with thermal resistance, the Sierpinski carpet, an exact self-similarity fractal, is used. Then, the equivalent electrical resistance network is constructed and the thermal conductivity of the porous media is derived by the thermalelectrical analogy technique.

Figure 1 displays zero- and one-stage Sierpinski carpets with $L=13$ and $C=5$. In figure 1, the white and black areas represent pores and particles, respectively. The fractal dimension for the Sierpinski carpet can also be expressed as [26]

$$
D_{\mathrm{f}}=\frac{\ln \left(L^{2}-C^{2}\right)}{\ln (L)} .
$$

The porosity of the Sierpinski carpet can be obtained as

$$
\varphi=\left(\frac{L^{2}-C^{2}}{L^{2}}\right)^{n+1},
$$

where the superscript $n(=0,1,2, \ldots)$ represents the stage of the Sierpinski carpet, $C$ is the cutout size and $L$ is the side length of the Sierpinski carpet. It is seen from figure 1 that if the cutout size, $C$ (or side length $L$ ), is changed, fractals with different fractal dimensions and different porosities can be obtained. Equation (10) also tells us that the porosity changes with $n$ even if $L$ and $C$ remain unchanged. Therefore, real porous media can be simulated by changing $C$ (or $L$ ) and $n$. In Ma et al's work [13], $L(=3)$ and $C(=1)$ are kept unchanged and so is the fractal dimension, $D_{\mathrm{f}}(=1.89)$, and in their work only $n$ is changed ( $n=6$ and 7 were used). Therefore, Ma et al's model is restricted to the porosity range of about $0.30-0.50$ (see figure 2). According to figure 2, at higher and lower porosities, the fractal dimension, $D_{\mathrm{f}}$, deviates much from 1.89. In order to predict accurately the thermal conductivity, a better model is desirable. After several trials, we found that the side length of the Sierpinski carpet $L=13$ is a proper choice for modelling real porous media and for covering a wider range of porosity. Then, we only need to choose the cutout size, $C$, and the stage, $n$, for different fractal dimensions and different porosities based on figure 2 or equations (9) and (10), see table 1 . Table 1 lists the fractal dimension $D_{\mathrm{f}}$ and porosity $\varphi$ at different length scales $(C / L)$ and stages $n$ based on equations (9) and (10). It is evident that the present model is different from that of Ma et al [13]. 


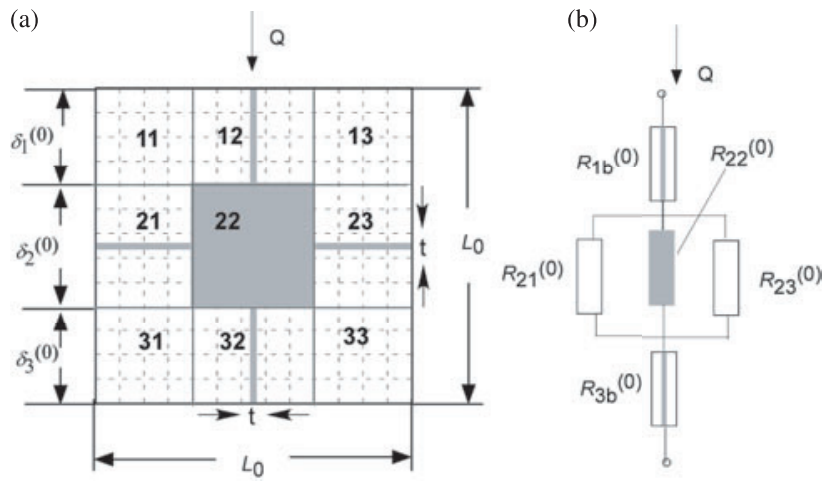

Figure 3. The thermal conductivity model and the thermal-electrical analogy for a zero-stage carpet with $L=13$ and $C=5$ : (a) the thermal conductivity model for a zero-stage carpet and $(b)$ the network of the thermal-electrical analogy for the zero-stage carpet. (a)

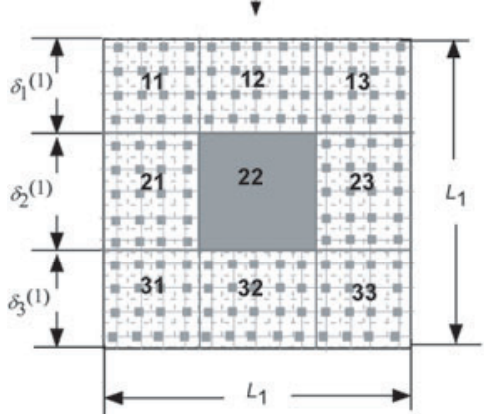

(b)

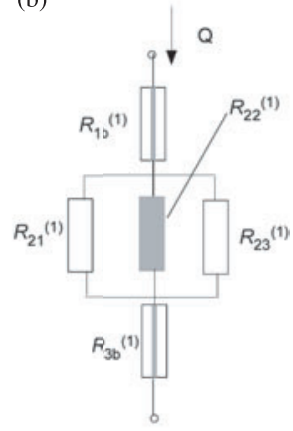

Figure 4. The thermal conductivity model and the thermal-electrical analogy for a one-stage carpet with $L=13$ and $C=5$ : $(a)$ the thermal conductivity model for a one-stage carpet and $(b)$ the network of the thermal-electrical analogy for the one-stage carpet.

Now, let us derive the generalized model for the effective thermal conductivity of porous media based on the selfsimilarity existing in porous media and based on the model shown in figure 1. In this work, the one-dimensional heat conduction model is again used to solve the thermal conductivity of porous media, the same as that adopted by Zehnder and Schlunder [3], Crane and Vachon [5], Hsu et al [10], Yu and Cheng [12] and Ma et al [13]. For justification of the one-dimensional heat conduction model, readers may consult the work of Ma et al [13].

Figures 3 and 4 show the heat conduction model for zero-stage and one-stage Sierpinski carpets, respectively. Figures $3(b)$ and $4(b)$ display the networks of the thermalelectrical analogy for the zero- and one-stage Sierpinski carpets, respectively. In figures 3 and 4, the bars represent the contact thermal resistances between particles. The dimensionless contact thermal resistance is defined as $t^{+}=$ $t / L$. It is expected that the contact thermal resistance between particles depends on the bar width, $t$. Since the Sierpinski carpet is a self-similar fractal geometry, the bar widths are also assumed to be self-similar. Ma et al [13] demonstrated that the lateral contact resistance has the negligible effect on the total thermal conductivity when the dimensionless contact thermal resistance $t^{+}(=t / L)<0.005$ in their Sierpinski carpet model with $C / L=1 / 3$. To examine this point in the present situation of $L=13$, the thermal conductivity of the horizontal-central part in figure 3(a) is calculated, see figure 5(a). In figure 5(a), the lateral contact thermal resistance is represented by the bars in the horizontal-central part and $C$ is the thickness of the horizontal-central part, $\delta_{2}$ (see figure 3 ). Figure $5(b)$ is the electrical analogy diagram. In figure $5(b), R_{21}, R_{22}$ and $R_{23}$ represent the thermal resistances, and the subscript 21, 22 and 23 denote the blocks marked 21, 22 and 23, respectively. The blocks marked 21, 22 and 23 are in parallel, but the blocks 21 and 23 are in series with bars, respectively. The resistance of block 21 is

$$
\begin{aligned}
R_{21} & =\frac{t}{k_{\mathrm{s}}(L-C) / 2}+\frac{C-t}{k_{\mathrm{f}}(L-C) / 2} \\
& =\frac{2}{k_{\mathrm{f}}(1-C / L)}\left[\frac{t^{+}}{\beta}+\frac{C}{L}-t^{+}\right]=R_{23},
\end{aligned}
$$

where $(L-C) / 2$ is the width of block $21, \beta$ is the ratio of thermal conductivities, $k_{\mathrm{s}} / k_{\mathrm{f}}$, and $t^{+}=t / L$.

The thermal resistance, $R_{2}$, of the three parallel parts gives

$$
R_{2}=\frac{R_{21} R_{22} R_{23}}{R_{21} R_{22}+R_{22} R_{23}+R_{23} R_{21}},
$$

where $R_{22}=1 / k_{\mathrm{s}}$.

The heat flow is defined by

$$
Q=\frac{T_{1}-T_{2}}{R_{2}}=k_{2, \mathrm{e}} \frac{T_{1}-T_{2}}{C} A,
$$

where $A=L \times 1, k_{2, \mathrm{e}}$ is the effective thermal conductivity of the horizontal-central part and $T_{1}-T_{2}$ is the temperature difference imposed on the unit cell or Sierpinski carpet. Then, the dimensionless effective thermal conductivity, $k_{2, \mathrm{e}}^{+}$, can be obtained as

$$
k_{2, \mathrm{e}}^{+}=\frac{k_{2, \mathrm{e}}}{k_{\mathrm{f}}}=\frac{1-C / L}{L(1 / \beta-1) t^{+} / C+1}+\frac{C}{L} \beta .
$$

Equation (14) indicates that when $t=C$, i.e. $t^{+}=t / L=C / L$, $k_{2, \mathrm{e}}^{+}=\beta$, which is expected because this situation corresponds to that of the material completely consisting of solid, and that when $t=0$, i.e. $t^{+}=0, k_{2, \mathrm{e}}^{+}=1+C(\beta-1) / L$, which is also expected because this situation corresponds to that of the material consisting of three parts (with perfect contact), solid particle (in the centre) and matrices (located lateral to the solid particle). Equation (14) depicts that the effective thermal conductivity of the horizontal-central part depends on the lateral contact thermal resistance, $t^{+}, \beta$ and the ratio $(=C / L)$ of geometrical length scales. Tables 2-3 demonstrate the effect of the lateral contact thermal resistance, $t^{+}$, on the total effective thermal conductivity of the structure as shown in figure $5(a)$ at different ratios $\beta$, based on equation (14). From table 2, it can be found that for the structure $C / L=3 / 13$, the error caused by the lateral contact thermal resistance $t^{+}=0.0030$ at $\beta=0.1$ is about $10 \%$ compared with $t^{+}=0$ at $\beta=0.1$. This level of error of about $10 \%$ is chosen to be acceptable. When $\beta \geqslant 0.1$, the effect of the lateral contact thermal resistance on the total thermal conductivity is negligible. This means that the effect of the lateral contact thermal resistance on a the total thermal conductivity becomes important only when 
(a)

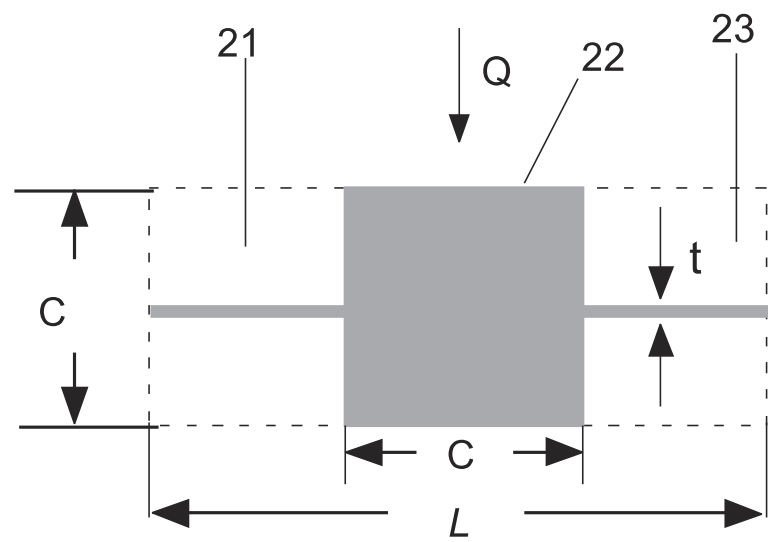

(b)

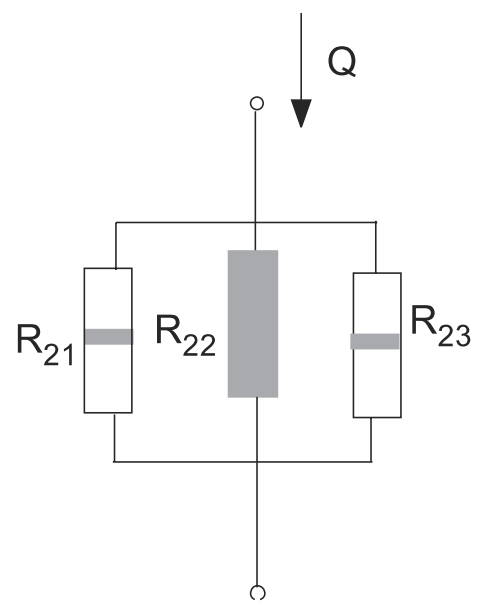

(c)

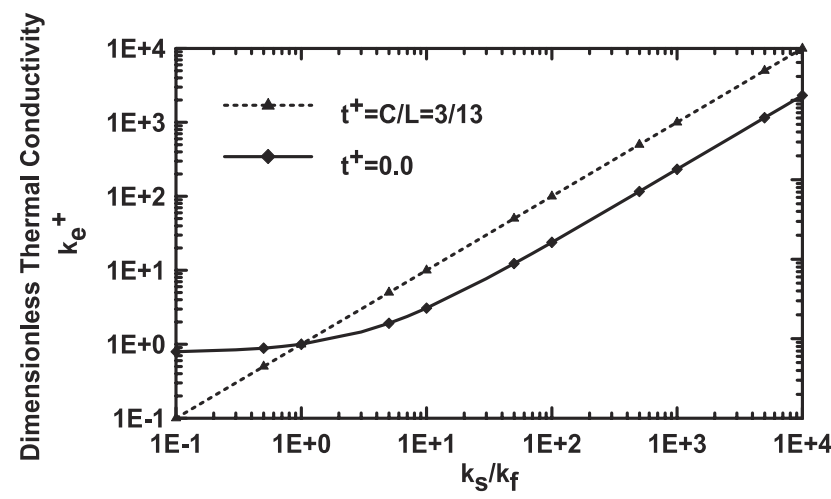

(d)

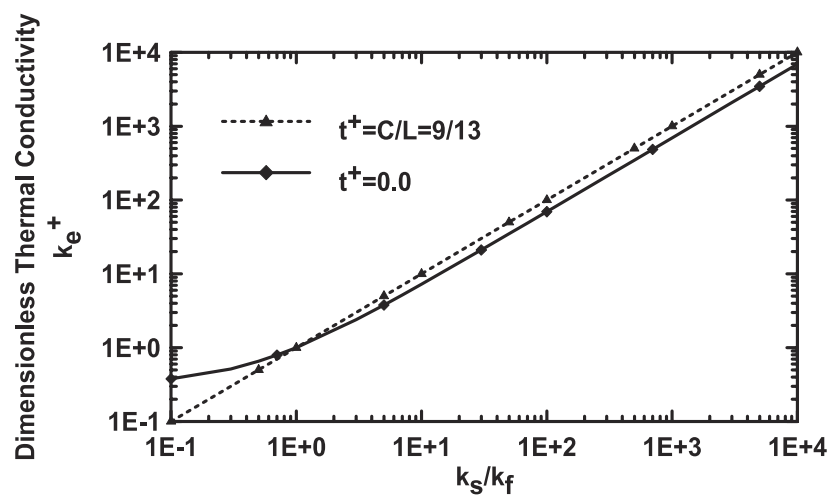

Figure 5. The effect of the lateral contact resistance on the thermal conductivities: (a) the thermal conductivity model for the horizontal-central part in figure $1(a) ;(b)$ the network of the thermal-electrical analogy for the horizontal-central part; $(c)$ thermal conductivity results when $L=13, C=3$ at different $t^{+}$; and $(d)$ thermal conductivity results when $L=13, C=9$ at different $t^{+}$.

Table 1. Fractal dimension, $D_{\mathrm{f}}$, and porosity $\varphi$, at different geometrical length scales $(C / L)$ and stages $(n)$.

\begin{tabular}{lllll}
\hline$C / L$ & $3 / 13$ & $5 / 13$ & $7 / 13$ & $9 / 13$ \\
$D_{\mathrm{f}}$ & 1.979 & 1.938 & 1.866 & 1.746 \\
$\varphi$ & 0.80 & 0.61 & 0.36 & 0.14 \\
$n$ & 2 & 2 & 2 & 3 \\
\hline
\end{tabular}

$t^{+}>0.0030$. In other words, if the lateral contact thermal resistance $t^{+}<0.0030$ at $C / L=3 / 13$, the effect of the lateral contact thermal resistance on the total thermal conductivity may be negligible. Similar results can be found from table 3; i.e. if the lateral contact thermal resistance $t^{+}<0.0090$ at $C / L=9 / 13$, the effect of the lateral contact thermal resistance on the total thermal conductivity may be neglected. This result tells us that if we choose the contact thermal resistance properly, the effect of the lateral contact thermal resistance on the total thermal conductivity can be neglected, and then the computation/analysis of the total thermal resistance and the total thermal conductivity of the whole structure, the Sierpinski carpet, can be simplified because the lateral contact thermal resistance may be not included in the computation/analysis. From our computations, the minimum lateral contact thermal resistance, $t_{\min }^{+}$(defined as that at which the error caused by the lateral contact thermal resistance is about $10 \%$, compared with perfect contact, $t^{+}=0$ at $\beta=0.1$ ) is correlated as

$$
t_{\min }^{+}=0.013 \times \frac{C}{L} .
$$

The results for the minimum lateral contact thermal resistance at the different geometrical length scales and the errors are summarized in table 4. From table 4 , it can be found that $t_{\min }^{+}$increases with the cutout size, $C$. This is expected and is consistent with the physical situations.

Figures $5(c)$ and $(d)$ show the general trends. It can be found that as $\beta>1$, the dimensionless thermal conductivities increase with the lateral contact thermal resistance (bar width) $t^{+}$. The opposite phenomenon is observed as $\beta<1$. This can be interpreted as that when $\beta>1$, the thermal conductivity of the solid is greater than that of the fluid (matrix), i.e. $k_{\mathrm{s}}>k_{\mathrm{f}}$. As the bar width increases, this corresponds to the increase of the portion of solid with high thermal conductivity, leading to the increase of the effective thermal conductivity. In the limit of $t^{+}=t / L=C / L$, the dimensionless thermal conductivities reach the maximum possible value. This is expected because when $\beta>1$, the unit cell as shown 
Table 2. Dimensionless thermal conductivity, $k_{2, \mathrm{e}}^{+}$, versus the dimensionless contact thermal resistance, $t^{+}$, and ratio $\beta$ of component thermal conductivities at $C / L=3 / 13$.

\begin{tabular}{lllllll}
\hline$\beta$ & 0.1 & 1 & 10 & 100 & 1000 & 10000 \\
$t^{+}=0$ & 0.7923 & 1.0 & 3.0769 & 23.8462 & 231.5385 & 2308.4614 \\
$t^{+}=0.0030$ & 0.7117 & 1.0 & 3.0860 & 23.8562 & 231.5486 & 2308.4717 \\
\hline
\end{tabular}

Table 3. Dimensionless thermal conductivity, $k_{2, \mathrm{e}}^{+}$, versus the dimensionless contact thermal resistance, $t^{+}$, and ratio $\beta$ of component thermal conductivities at $C / L=9 / 13$.

\begin{tabular}{lllllll}
\hline$\beta$ & 0.1 & 1 & 10 & 100 & 1000 & 10000 \\
$t^{+}=0$ & 0.3769 & 1.0 & 7.2308 & 69.5385 & 692.6154 & 6923.3848 \\
$t^{+}=0.0090$ & 0.3447 & 1.0 & 7.2344 & 69.5425 & 692.6194 & 6923.3887 \\
\hline
\end{tabular}

Table 4. The minimum lateral contact thermal resistance, $t_{\min }^{+}$, with different geometrical length scales $(C / L)($ at $\beta=0.1)$.

\begin{tabular}{lllll}
\hline$C / L$ & $3 / 13$ & $5 / 13$ & $7 / 13$ & $9 / 13$ \\
$t_{\min }^{+}$ & 0.0030 & 0.0050 & 0.0070 & 0.0090 \\
Error \% & 10.17 & 9.86 & 9.38 & 8.55 \\
\hline
\end{tabular}

in figure $5(a)$ is completely filled with a solid material of high thermal conductivity, resulting in the highest thermal conductivity for the unit cell. When $\beta<1$, this means $k_{\mathrm{s}}<k_{\mathrm{f}}$. In this situation, as the bar width increases, this corresponds to the increase of the portion of solid with low thermal conductivity, leading to the decrease of the effective thermal conductivity. In the limit of $t^{+}=t / L=$ $C / L$, the dimensionless thermal conductivity reaches the minimum possible value, $\beta$ (as $\beta<1$ ). This is also expected for the same reason, i.e. in this situation the unit cell as shown in figure 5(a) is filled with material of low thermal conductivity, leading to the lowest effective thermal conductivity for the unit cell. From figures $5(c)$ and $(d)$ it can also be seen that when $t^{+}=0$ (i.e. perfect contact), the thermal conductivity increases with the ratio of $C / L$ when $\beta>1$ and decreases with the increase of the ratio $C / L$ when $\beta<1$. This is expected and is consistent with the physical situations for the same reason as above.

Since we have proved that the lateral contact resistance is not important if we choose $t^{+} \leqslant t_{\min }^{+}$, the longitudinal contact thermal resistance (defined as the contact thermal resistance along the heat flow direction) is only taken into account in the present model derived here. Under the assumption that the one-dimensional heat flow model is applicable under a steady state, the present model is derived as follows.

In figure $3(a), \delta_{1}^{(0)}\left(=\left(L^{(0)}-C^{(0)}\right) / 2\right), \delta_{2}^{(0)}(=C)$ and $\delta_{3}^{(0)}\left(=\delta_{1}^{(0)}\right)$ denote the thicknesses of the different layers along the heat flow through the zero-stage Sierpinski carpet. Figure $3(b)$ shows the electrical analogy diagram for the zero-stage Sierpinski carpet, and the effect of the lateral contact thermal resistance is ignored in the calculation and only the contact thermal resistance, $R_{1 \mathrm{~b}}^{(0)}$, along the heat flow direction is considered. The thermal resistance, $R_{1}^{(0)}$, of layer 1 is

$$
\begin{aligned}
R_{1}^{(0)} & =\frac{R_{1} R_{1 \mathrm{~b}}^{(0)}}{R_{1}+R_{1 \mathrm{~b}}^{(0)}}=\frac{\left(L^{(0)}-C^{(0)}\right) / 2}{k_{\mathrm{f}}(1-t)+k_{\mathrm{s}} t} \\
& =\frac{L^{(0)}-C^{(0)}}{2 k_{\mathrm{f}} L^{(0)}\left[\left(1-t^{+}\right)+\beta^{(0)} t^{+}\right]},
\end{aligned}
$$

where the superscript ' 0 ' refers to the zero-stage Sierpinski carpet, $\beta^{(0)}=k_{\mathrm{s}} / k_{\mathrm{f}}$ and $R_{1}$ is the thermal resistance of the fluid phase material of layer 1 .

The thermal resistance, $R_{2}^{(0)}$, of layer 2 is considered to be the parallel equivalent of the materials marked 21, 22 and 23 in figure $3(a)$, and it is

$$
\begin{aligned}
R_{2}^{(0)} & =\frac{R_{21}^{(0)} R_{22}^{(0)} R_{23}^{(0)}}{R_{21}^{(0)} R_{22}^{(0)}+R_{22}^{(0)} R_{23}^{(0)}+R_{23}^{(0)} R_{21}^{(0)}} \\
& =\frac{C^{(0)}}{k_{\mathrm{f}}\left[\left(L^{(0)}-C^{(0)}\right)+\beta^{(0)} C^{(0)}\right]} .
\end{aligned}
$$

Due to the symmetry, the thermal resistance, $R_{3}^{(0)}$, of layer 3 is

$$
R_{3}^{(0)}=\frac{R_{3} R_{3 \mathrm{~b}}^{(0)}}{R_{3}+R_{3 \mathrm{~b}}^{(0)}}=R_{1}^{(0)} .
$$

The total thermal resistance, $R_{\mathrm{sc}}^{(0)}$, of the zero-stage Sierpinski carpet is considered as the series equivalent of layers 1,2 and 3, and is

$$
\begin{aligned}
R_{\mathrm{sc}}^{(0)} & =R_{1}^{(0)}+R_{2}^{(0)}+R_{3}^{(0)} \\
& =\frac{1}{k_{\mathrm{f}}}\left(\frac{1-C^{(0)} / L^{(0)}}{t^{+}\left(\beta^{(0)}-1\right)+1}+\frac{C^{(0)}}{C^{(0)}\left(\beta^{(0)}-1\right)+L^{(0)}}\right) .
\end{aligned}
$$

Thus the dimensionless effective thermal conductivity, $k_{\mathrm{e}, \mathrm{sc}}^{+(0)}$, for the zero-stage Sierpinski carpet can be obtained as

$$
\begin{aligned}
k_{\mathrm{e}, \mathrm{sc}}^{+(0)} & =\frac{k_{\mathrm{e}, \mathrm{sc}}^{(0)}}{k_{\mathrm{f}}}=\frac{L^{(0)}}{R_{\mathrm{sc}}^{(0)} A_{0} k_{\mathrm{f}}} \\
& =\left(\frac{1-C^{(0)} / L^{(0)}}{t^{+}\left(\beta^{(0)}-1\right)+1}+\frac{C^{(0)}}{C^{(0)}\left(\beta^{(0)}-1\right)+L^{(0)}}\right)^{-1},
\end{aligned}
$$

where $A_{0}=L^{(0)} \times 1$ and $L^{(0)}$ is the side length of the zero-stage Sierpinski carpet.

A similar method can be used to derive the effective thermal conductivity of a one-stage carpet (see figure 4(a)). In figure 4(a), except for the central shaded region with conductivity, $k_{\mathrm{s}}$, the other regions are considered as an equivalent homogeneous material with thermal conductivity $k_{\mathrm{e}, \mathrm{sc}}^{(0)}$. Thus, the dimensionless effective thermal conductivity, $k_{\mathrm{e}, \mathrm{sc}}^{+(1)}$, of the one-stage carpet is

$$
\begin{aligned}
k_{\mathrm{e}, \mathrm{sc}}^{+(1)} & =k_{\mathrm{e}, \mathrm{sc}}^{+(0)} \\
& \times\left(\frac{1-C^{(1)} / L^{(1)}}{t^{+}\left(\beta^{(1)}-1\right)+1}+\frac{C^{(1)}}{C^{(1)}\left(\beta^{(1)}-1\right)+L^{(1)}}\right)^{-1},
\end{aligned}
$$

where $\beta^{(1)}=\beta^{(0)} / k_{\mathrm{e}, \mathrm{sc}}^{+(0)}$. 
For an $n$-stage carpet, the dimensionless effective thermal conductivity is

$$
\begin{aligned}
k_{\mathrm{e}, \mathrm{sc}}^{+(n)} & =k_{\mathrm{e}, \mathrm{sc}}^{+(n-1)} \\
& \times\left(\frac{1-C^{(n)} / L^{(n)}}{t^{+}\left(\beta^{(n)}-1\right)+1}+\frac{C^{(n)}}{C^{(n)}\left(\beta^{(n)}-1\right)+L^{(n)}}\right)^{-1},
\end{aligned}
$$

where $\beta^{(n)}=\beta^{(0)} / k_{\mathrm{e}, \mathrm{sc}}^{+(n-1)}$ and the superscript $n=1,2, \ldots$ Equation (22) presents a recursive algorithm for the thermal conductivity of self-similar porous media.

Since this model assumes that the porous media consist of two parts, randomly distributed non-touching particles and self-similarly distributed particles contacting each other with thermal resistance between them, these two portions are considered to be in parallel. The total thermal resistance, $R_{\mathrm{t}}$, can thus be expressed as

$$
\frac{1}{R_{\mathrm{t}}}=\frac{1}{R_{\mathrm{nt}}}+\frac{1}{R_{\mathrm{sc}}},
$$

where the subscripts $t$, nt and sc represent the total, nontouching and Sierpinski carpets, respectively. The effective thermal conductivity, $k_{\mathrm{e}}$, of the system can be obtained as

$$
\begin{aligned}
k_{\mathrm{e}}= & \frac{1}{R_{\mathrm{t}}} \frac{L}{A}=\frac{1}{R_{\mathrm{nt}}} \frac{L}{A}+\frac{1}{R_{\mathrm{sc}}} \frac{L}{A}=\frac{1}{R_{\mathrm{nt}}} \frac{L}{A_{\mathrm{nt}}} \frac{A_{\mathrm{nt}}}{A}+\frac{1}{R_{\mathrm{sc}}} \frac{L}{A_{\mathrm{sc}}} \frac{A_{\mathrm{sc}}}{A} \\
= & \frac{A_{\mathrm{nt}}}{A} k_{\mathrm{e}, \mathrm{nt}}+\left(1-\frac{A_{\mathrm{nt}}}{A}\right) k_{\mathrm{e}, \mathrm{sc}},
\end{aligned}
$$

where $A$ is the total area of a representative cross section and $A_{\text {nt }}$ is an equivalent area of a cross section having the same porosity as the non-touching particles, with $0 \leqslant A_{\text {nt }} / A \leqslant 1$ and $A_{\mathrm{sc}} / A=1-A_{\mathrm{nt}} / A$. For non-touching particles, Hsu et al [10] obtained the following thermal conductivity expression,

$$
k_{\mathrm{e}, \mathrm{nt}}=k_{\mathrm{f}}(1-\sqrt{1-\varphi})+\frac{k_{\mathrm{f}} \sqrt{1-\varphi}}{1+(1 / \beta-1) \sqrt{1-\varphi}},
$$

which will be used in connection with the first term of the right-hand side of equation (24).

Substituting equations (22) and (25) into equation (24) gives the following expression for the dimensionless effective thermal conductivity, $k_{\mathrm{e}}^{+}$, of porous media:

$$
\begin{aligned}
k_{\mathrm{e}}^{+}= & \frac{k_{\mathrm{e}}}{k_{\mathrm{f}}}=\frac{A_{\mathrm{nt}}}{A} k_{\mathrm{e}, \mathrm{nt}}^{+}+\left(1-\frac{A_{\mathrm{nt}}}{A}\right) k_{\mathrm{e}, \mathrm{sc}}^{+} \\
= & \frac{A_{\mathrm{nt}}}{A}\left[(1-\sqrt{1-\varphi})+\frac{\sqrt{1-\varphi}}{1+(1 / \beta-1) \sqrt{1-\varphi}}\right] \\
& +\left(1-\frac{A_{\mathrm{nt}}}{A}\right) k_{\mathrm{e}, \mathrm{sc}}^{+(n)},
\end{aligned}
$$

where the superscript $n=1,2, \ldots, k_{\mathrm{e}, \mathrm{nt}}^{+}=k_{\mathrm{e}, \mathrm{nt}} / k_{\mathrm{f}}$, and $k_{\mathrm{e}, \mathrm{sc}}^{+(n)}$ is given by equation (22).

The recursive algorithm for the effective conductivity is summarized as follows:

(a) The values of $\beta^{(0)}\left(=k_{\mathrm{s}} / k_{\mathrm{f}}=\beta\right.$, in this paper $\left.\beta=0.1-10^{4}\right)$, the side length, $L(=13)$, of the Sierpinski carpet, the particle size, $C$, porosity, $\varphi$, contact parameter, $t^{+}$, and area ratio, $A_{n} / A$, are given.

(b) Calculate the fractal dimension, $D_{\mathrm{f}}$, and the stage, $n$, from equations (9) and (10), respectively.

(c) Calculate the dimensionless effective thermal conductivity, $k_{\mathrm{e}, \mathrm{sc}}^{+(0)}$, of a zero-stage carpet from equation (20).

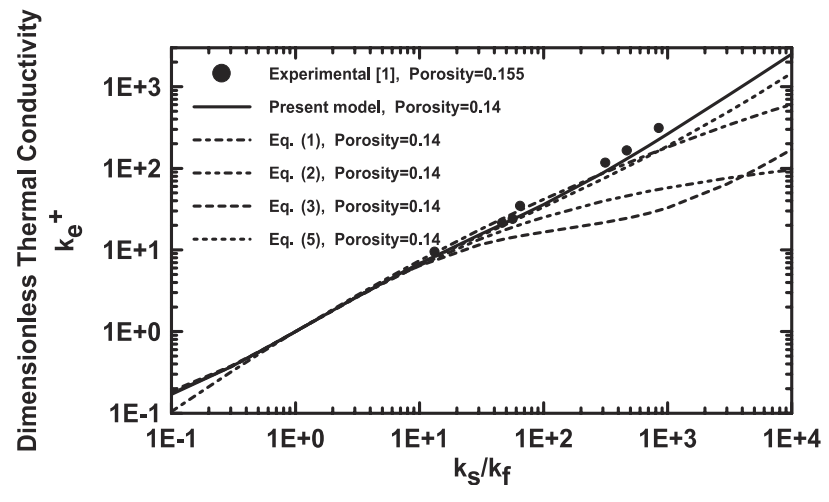

Figure 6. A comparison of the present model predictions with the existing experimental data and predictions by other models at a porosities $0.14-0.155$.

(d) Calculate the value of $\beta^{(n)}\left(=\beta^{(0)} / k_{\mathrm{e}, \mathrm{sc}}^{+(n-1)}, n=\right.$ $1,2,3, \ldots)$.

(e) Find the dimensionless effective thermal conductivity, $k_{\mathrm{e}, \mathrm{sc}}^{+(n)}$, from equation (22) for the self-similar porous medium.

(f) Find the dimensionless effective thermal conductivity, $k_{\mathrm{e}}^{+}$, from equation (26) for the porous medium.

Procedures (d) and (e) are repeated up to the required Sierpinski carpet's stage, $n$, for the dimensionless effective thermal conductivity of the self-similar porous medium. Once $k_{\mathrm{e}, \mathrm{sc}}^{+(n)}$ is obtained, $k_{\mathrm{e}}^{+}$can be found from procedure (f). It is seen that the present algorithm for the thermal conductivity is quite simple, and it takes less than $1 \mathrm{~s}$ in a microcomputer for one configuration ( $\beta=0.1-10^{4}$ at a given porosity).

\section{Results and discussion}

In this work, Sierpinski carpets with constant $L=13$ and different cutout size $C=3,5,7$ and 9 are selected to simulate porous media with different porosities. The pore area fractal dimensions for the four Sierpinski carpets $\operatorname{are} D_{\mathrm{f}}=1.979$, $1.938,1.866$ and 1.746, respectively. The four Sierpinski carpets are used to model real porous media with porosity ranges of $\varphi>0.80, \varphi=0.60-0.80, \varphi=0.30-0.60$ and $\varphi<0.30$, respectively, by choosing a different stage, $n$.

Figure 6 presents a comparison among the present model predictions $\left(A_{\mathrm{nt}} / A=0.10\right.$ and $t^{+}=0.0090$ are chosen for matching the experimental data) by the Sierpinski carpet ( $L=13, C=9, D_{\mathrm{f}}=1.746$, at stage $n=2$ and porosity $0.14)$, existing experimental data at porosity 0.155 and other models (equations (1)-(3) and (5)). In Hsu et al's model, equation (3) for a mono-dispersed porous medium, $\gamma_{\mathrm{c}}=0.13$, is used. In Ma et al's model, equation (5), $A_{\mathrm{nt}} / A=0.55$, and $t^{+}=0.00033$ are taken. It is seen that the predicted thermal conductivities of the present model are in good agreement with the experimental data of Woodside and Messmer [1]. However, the other models present under-estimated values when the ratio of solid to fluid conductivity is higher (than 10 for equations (2) and (3), and than 200 for equation (1)). On the other hand, Ma et al's model, equation (5), gives a lower estimation when the ratio of solid to fluid conductivity is higher than 100 . It should be noted that in figure 6 the comparisons are made 


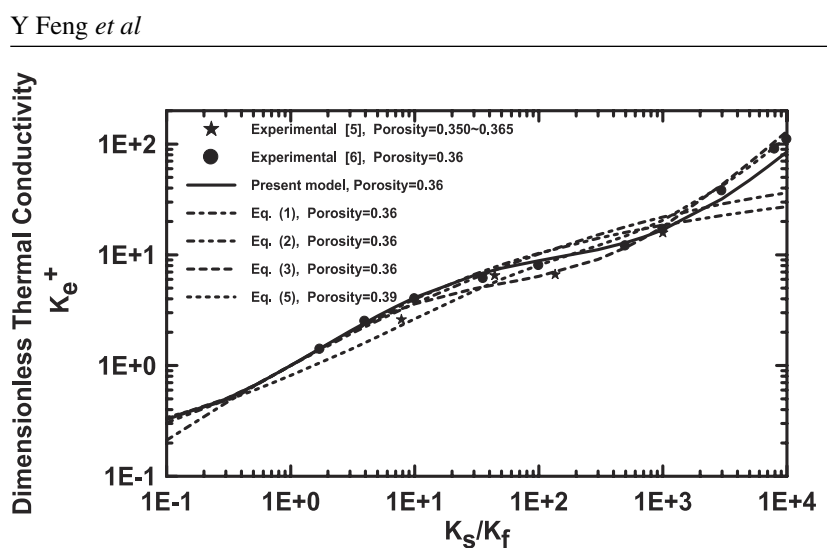

Figure 7. A comparison of the present model predictions with the existing experimental data and predictions by other models at a porosity of 0.36 .

at a porosity of 0.155 for experimental data and at a porosity of 0.14 for theoretical models. The reason is that the porosity of the present theoretical model is related to the stage, $n$, of the Sierpinski carpet by equation (10), and the stage, $n$, is an integer. When we take the Sierpinski carpet with $L=13$, $C=9, D_{\mathrm{f}}=1.746$ and $n=2$, the porosity is $\varphi=0.14$. Since the experimental data on thermal conductivity exactly at porosity 0.14 are not available in the literature and the available experimental data at the porosity closest to 0.14 are those at 0.155 , we have to choose the experimental data at porosity 0.155 for comparison.

Figure 7 compares the predictions of the present model with the existing experimental data and the predictions of other models at porosity 0.36. In the predictions of the present model, the Sierpinski carpet with $L=13, C=7$ and $D_{\mathrm{f}}=1.866$ at stage $n=2$ is used. The parameters $A_{\mathrm{nt}} / A=0.10$ and $t^{+}=0.00050$ are chosen in the calculation of the thermal conductivity for matching the experimental data. Good agreement is found between the model predictions and the experimental data. It is also found that the model predictions of the present model almost have the same accuracy as Hsu et al's model, equation (3), and Ma et al's model, equation (5). This is expected because, as mentioned in the previous section, Ma et al's model is valid only in the porosity range $0.3-0.5$, and Hsu et al's model may also be valid only at a porosity of around 0.40 if $\gamma_{c}=0.13$ is taken. However, equations (1) and (2) under-predict the stagnant thermal conductivity when $\beta>2 \times 10^{3}$. It should also be noted that in figure 7 a porosity of 0.39 is applied when equation (5) is used. The reason is that since equation (5) is for the Sierpinski carpet with $L=3, C=1$ and $n=6$, according to equation (10), the porosity closest to 0.36 is 0.39 when $n=6$. Therefore, we have to choose a porosity of 0.39 when comparing Ma et al's model, equation (5), with the available experimental data at porosity around 0.36 .

Figure 8 is a comparison among the present model predictions (by the Sierpinski carpet with $L=13, C=5$ and $n=2$ ), the experimental data and predictions by other models. In the present model the parameters $A_{\mathrm{nt}} / A=0.10$ and $t^{+}=$ 0.00050 are again chosen for matching the experimental data. From figure 8, it can be seen that the thermal conductivities predicted by the present model are in good agreement with the experimental data of Verma et al [8] and Misra et al [9].

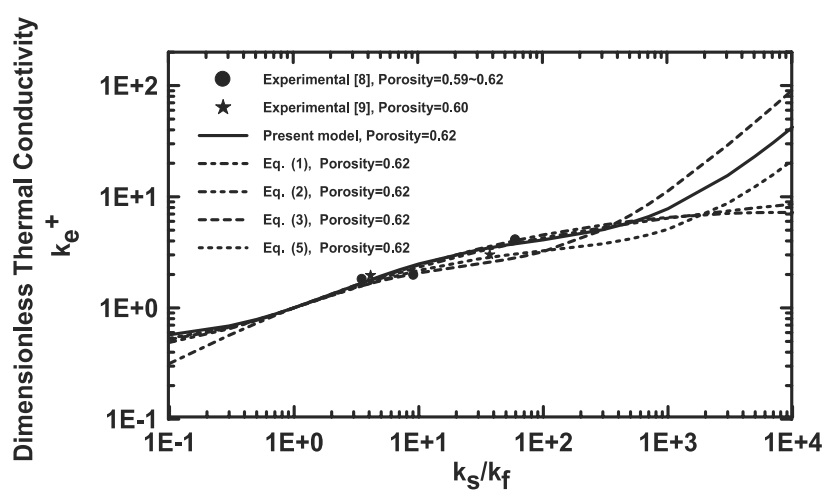

Figure 8. A comparison of the present model predictions with the existing experimental data and predictions by other models at a porosity of 0.62 .

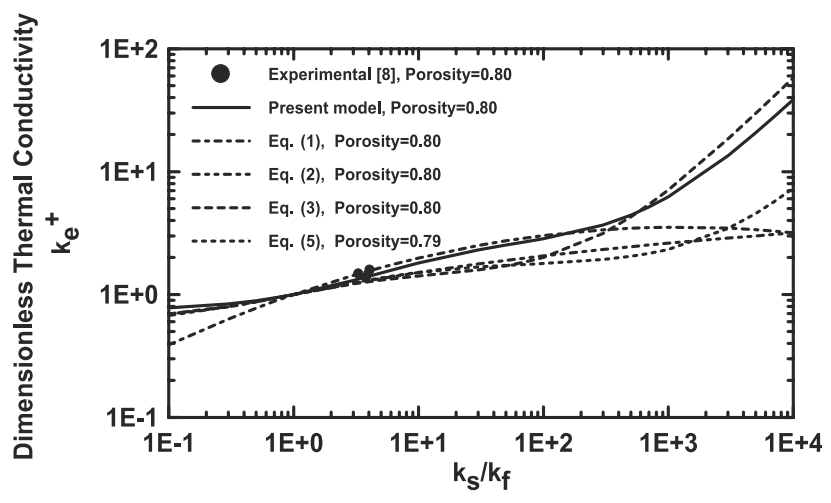

Figure 9. A comparison of the present model predictions with the existing experimental data and predictions by other models at a porosity of 0.80 .

Whereas equations (1) and (2) again predict lower values of the thermal conductivities when the ratio of solid to fluid conductivity is higher than 400, Hsu et al's model predicts a lower thermal conductivity when the ratio of solid to fluid conductivity is higher than 10 , but a higher thermal conductivity when the ratio of solid to fluid conductivity is higher than 400 .

Figure 9 shows the model predictions, which also agree well with the existing experimental data at a porosity of 0.80 . The Sierpinski carpet with $L=13, C=3, D_{\mathrm{f}}=1.979$ and $n=3$ is used in the calculation. The parameters $A_{\mathrm{nt}} / A=0.10$ and $t^{+}=0.00050$ are again used in the calculation for matching the experimental data. The same reason as that given for figure 7 can be used to explain figure 9 , in which the porosity of 0.79 is calculated from the model equation (5) for the Sierpinski carpet with $L=3, C=1$, $n=1$. The porosity of 0.79 is the closest to 0.80 , at which the experimental data are taken from [8]. Therefore, in Ma et al's model, a porosity of 0.79 is applied for comparison. Since no other experimental data on the thermal conductivities of porous media are available, no extensive comparison is given in figure 9. But similar trends can still be found from figure 9, i.e. equations (1) and (2) predict lower values of the thermal conductivities when the ratio of solid to fluid conductivity is higher than 300, and Ma et al's model also predicts a lower thermal conductivity when the ratio of solid to fluid conductivity is higher than 1 , while Hsu et al's model 


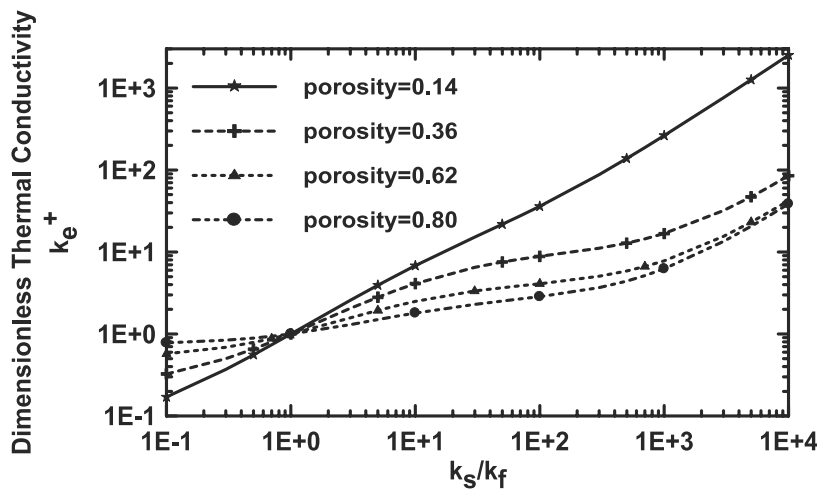

Figure 10. The effect of porosity on the effective thermal conductivity of porous media.

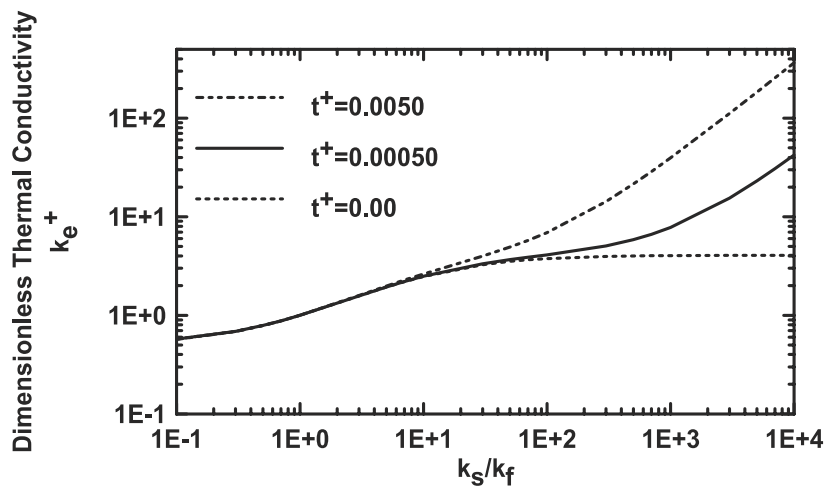

Figure 11. The effect of the longitudinal contact resistance, $t^{+}$, on the effective thermal conductivity at a porosity of 0.62 with $L=13$, $C=5, D_{\mathrm{f}}=1.938$ and $n=2$.

gives a lower thermal conductivity when the ratio of solid to fluid conductivity is higher than 5 but gives a higher thermal conductivity when the ratio of solid to fluid conductivity is higher than 500 .

The effect of porosity on the thermal conductivity of porous media is shown in figure 10. Figure 10 indicates that the thermal conductivity decreases with increasing porosity when $\beta>1$. This can be interpreted as that when the porosity increases, the solid volume fraction with higher thermal conductivity decreases, leading to the decrease in the effective thermal conductivity. But when $\beta<1$, the opposite result is expected, i.e. the thermal conductivity will increase with porosity. This figure again shows that the present model presents reasonable results.

Figure 11 shows the effect of the longitudinal contact thermal resistance, $t^{+}$(defined as the contact thermal resistance along the heat flow direction), on the dimensionless effective thermal conductivity. It can be seen from the figure that the effective thermal conductivity is independent of the contact parameter, $t^{+}$, when $\beta<20$. This implies that when the ratio $\beta$ of component conductivities is low, the contact between particles can be considered to be perfect and the contact resistance is not important. As $\beta>20$, the effective thermal conductivity increases with the parameter $t^{+}$. This situation corresponds to that where the matrix and the contact thermal resistance, $t^{+}$, are in parallel, but the contact thermal resistance, $t^{+}$, and particle are in series, leading to the increase in the effective thermal conductivity with the parameter $t^{+}$.

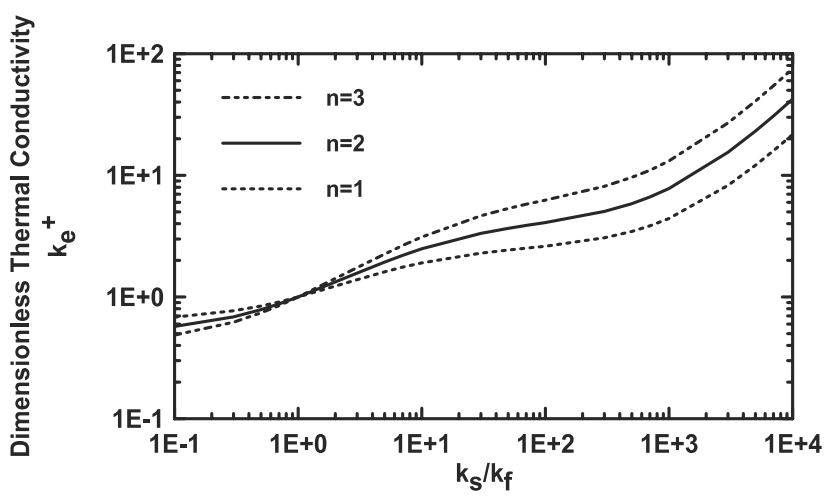

Figure 12. Effect of the stage, $n$, of the Sierpinski carpet on the effective thermal conductivity, with $L=13, C=5$ and $D_{\mathrm{f}}=1.938$.

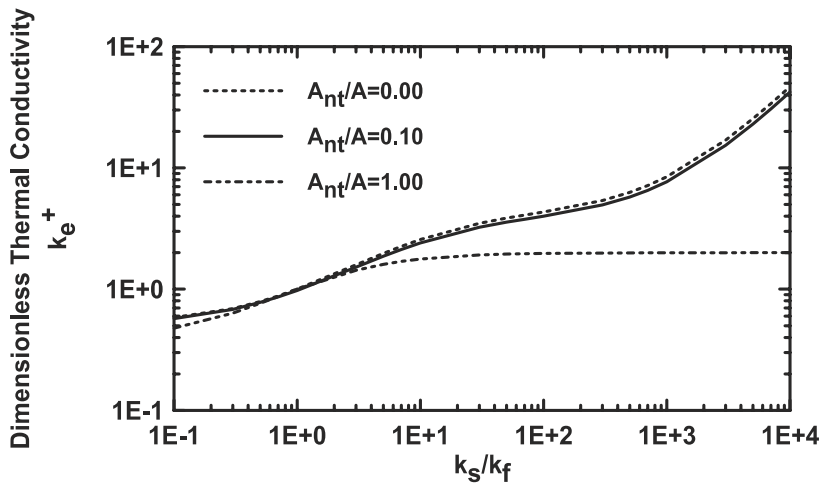

Figure 13. Effect of the area ratio on the effective thermal conductivity at porosity 0.62 with $L=13, C=5, D_{\mathrm{f}}=1.938$ and $n=2$.

This trend that the effective thermal conductivity increases with the parameter $t^{+}$is in qualitative agreement with Hsu et al's model [10].

Figure 12 depicts the effect of the stage, $n$, of the Sierpinski carpet (with $L=13, C=5, D_{\mathrm{f}}=1.938$ ) on the effective thermal conductivity. It is seen that the thermal conductivity increases as the stage, $n$, increases when $\beta>1$. This can be interpreted as that when the stage, $n$, increases, its porosity decreases and the solid volume fraction with higher thermal conductivity increases, leading to the increase in the effective thermal conductivity. But for $\beta<1$, the opposite result is observed, i.e. the thermal conductivity increases with a decrease in the stage, $n$.

The effect of the area ratio, $A_{\mathrm{nt}} / A$, on the effective thermal conductivity of the porous medium is shown in figure 13. For a fixed contact parameter $t^{+}=0.00050$ and stage $n=2$ of the Sierpinski carpet, the effective thermal conductivity is affected significantly by the area ratio $A_{\mathrm{nt}} / A$. From the figure, it is clear that when the ratio $A_{\mathrm{nt}} / A=1.0$, all particles are in the non-contact status, resulting in the minimum effective thermal conductivity. When the ratio $A_{\mathrm{nt}} / A=0$, all particles are in contact with each other, leading to the maximum effective thermal conductivity. The lower the ratio $A_{\text {nt }} / A$, the higher the fraction of particles in contact with each other, leading to the higher effective thermal conductivity. This is consistent with the physical situation. Therefore, figure 13 again shows that the present model presents reasonable results for the effective thermal conductivity. 


\section{Concluding remarks}

A generalized model for the thermal conductivity of porous media is derived based on the self-similarity existing in porous media and on the thermal-electrical analogy technique. Sierpinski carpets with the same side length $L=13$ but different cutout size $C(=3,5,7$ and 9) and different fractal dimensions are adopted for modelling real porous media in a wide range of porosities, $0.14-0.80$. The recursive algorithm is quite simple. The present model for the thermal conductivity of porous media is found to be a function of the porosity (related to the stage, $n$, of the Sierpinski carpet), the ratio $A_{\mathrm{nt}} / A$ of areas, the ratio $\beta$ of component thermal conductivities, contact thermal resistance, $t^{+}$, and microstructures $L$ and $C$. This model has the fewest parameters, $A_{\mathrm{nt}} / A$ and $t^{+}$, compared with the other models, and every parameter in this model has a clear physical meaning. The model predictions are compared with the existing experimental data and the predictions of other models, and the results show that the present model presents a good agreement with the existing experimental data in a wide range of porosities, $0.14-0.80$, and the present model is thus applicable for prediction of the thermal conductivity of real porous media.

\section{Acknowledgment}

This work was supported by the National Natural Science Foundation of China through project number 10272052.

\section{References}

[1] Woodside W and Messmer J H 1961 Thermal conductivity of porous media. II. Consolidated rocks J. Appl. Phys. 32 1699

[2] Krupiczka R 1967 Analysis of thermal conductivity in granular materials Int. Chem. Eng. 7122

[3] Zehnder P and Schlunder E U 1970 Thermal conductivity of granular materials at moderate temperatures Chemie. Ingr.-Tech. 42933 (in German)

[4] Hadley G R 1986 Thermal conductivity of packed metal powers Int. J. Heat Mass Transfer 29909

[5] Crane R A and Vachon R I 1977 A prediction of the bounds on the effective thermal conductivity of granular materials Int. J. Heat Mass Transfer 20711

[6] Nozad I, Carbonell R G and Whitaker S 1985 Heat conduction in multiphase systems-II Chem. Eng. Sci. 40857
[7] Prasad V, Kladias N, Bandyopadhaya A and Tian Q 1989 Evaluation of correlations for stagnant thermal conductivity of liquid-saturated porous beds of spheres Int. J. Heat Mass Transfer 321793

[8] Verma L S, Shrotriya A K, Ramvir S and Chaudhary D R 1991 Thermal conduction in two-phase materials with spherical and non-spherical inclusions J. Phys. D: Appl. Phys. 24 1729

[9] Misra K, Shrotriya A K, Singh R and Chaudhary 1994 Porosity correction for thermal conduction in real two-phase systems J. Phys. D: Appl. Phys. 27732

[10] Hsu C T, Cheng P and Wong K W 1995 A lumped-parameter model for stagnant thermal conductivity of spatially periodic porous media J. Heat Transfer 117264

[11] Kaviany M 1995 Principles of Heat Transfer in Porous Media (New York: Springer) p 147

[12] Yu B M and Cheng P 2002 Fractal models for the effective thermal conductivity of bi-dispersed porous media J. Thermophys. Heat Transfer 1622

[13] Ma Y T, Yu B M, Zhang D M and Zou M Q 2003 A self-similarity model for effective thermal conductivity of porous media J. Phys. D: Appl. Phys. 362157

[14] Mandelbrot B B 1982 The Fractural Geometry of Nature (New York: W H Freeman) p 23

[15] Feder J 1988 Fractals (New York: Plenum Press) pp 7-30, 184-92

[16] Katz A J and Thompson A H 1985 Fractal sandstone pores: implications for conductivity and pore formation Phys. Rev. Lett. 541325

[17] Krohn C E and Thompson A E 1986 Fractal sandstone pores: automated measurements using scanning-electronmicroscope images Phys. Rev. B 336366

[18] Thovert J F, Wary F and Adler P M 1990 Thermal conductivity of random media and regular fractals J. Appl. Phys. 683872

[19] Adler P M 1996 Transports in fractal porous media J. Hydrology 187195

[20] Smidt J M and Monro D M 1998 Fractal modeling applied to reservoir characterization and flow simulation Fractals $\mathbf{6}$ 401

[21] Yu B M, Lee L J and Cao H Q 2001 Fractal characters of pore microstructures of textile fabrics Fractals 9155

[22] Yu B M and Li J H 2001 Some fractal characters of porous media Fractals 9365

[23] Yu B M and Cheng P 2002 A fractal permeability model for bi-dispersed porous media Int. J. Heat Mass Transfer $\mathbf{4 5}$ 2983

[24] Yu B M, Lee L J and Cao H Q 2002 A fractal in-plane permeability model for fabrics Polym. Compos. 23201

[25] Yu B M and Liu W 2004 Fractal analysis of permeabilities for porous media AIChE J. $\mathbf{5 0} 57$

[26] Yu B M and Yao K L 1988 Critical percolation probalities for site problem on Sierpinski carpets Z. Phys. B: Condens. Matter 70209 